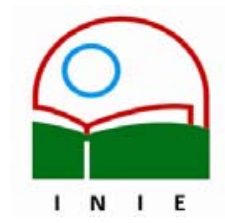

Universidad de Costa Rica

Facultad de Educación

Instituto de Investigación en Educación

ACTUALIDADES INVESTIGATIVAS EN EDUCACION

\title{
EL EXPEDIENTE ACUMULATIVO DEL NIÑO Y LA NIÑA, RECURSO PEDAGÓGICO EN EL NIVEL PREESCOLAR Y I Y II CICLOS DE LA EDUCACIÓN GENERAL BÁSICA
}

\begin{abstract}
Helvetia Cárdenas Leitón ${ }^{1}$
Resumen: El presente artículo describe una investigación que tuvo como fin analizar las funciones que cumple el expediente acumulativo del niño y la niña en el nivel de educación preescolar y l y II Ciclos. Se exponen las percepciones, prácticas y conocimientos de las profesoras de preescolar y I y II ciclos de la EGB y comités de orientación, la información se recoge mediante la técnica de grupos focales, cuestionario y revisión de expedientes acumulativos facilitados por las personas involucradas en la investigación. El análisis se realiza a la luz de la teoría de los expedientes acumulativos y, con ello, se llega a conclusiones y recomendaciones, entre las que se destaca una propuesta de expediente acumulativo que permite registrar información del niño y la niña de manera progresiva y sistemática, que a la vez, facilita el trabajo desde su ingreso, permanencia y egreso de un nivel a otro.
\end{abstract}

Palabras claves: EXPEDIENTE ACUMULATIVO/ SEGUIMIENTO INDIVIDUAL/ SISTEMATIZACIÓN DE DESARROLLO/ ATENCIÓN A LA DIVERSIDAD/ EDUCACIÓN PREESCOLAR/ EDUCACIÓN PRIMARIA/ EVALUACIÓN/

\begin{abstract}
The following article describes a research that had as main goal to analyze the functions that are performed by the accumulative file of boys and girls in preschool and I and II cycles. This research exposes the perceptions, practices, and knowledge of the teachers in preschooler and I and II cycles of the EGB, and orientation committees. The information is collected by means of the focal group technique, and by reviewing the accumulative files facilitated by people concerned in the study. The analysis is carried out based on the theory of the accumulative files and by this means, comes the conclusions, and recommendations, among those a proposal of accumulative file which allows the teacher to register boys and girls' information in a progressive and systematic form stands out, which at the same time, facilitates the work from the moment it begins, permanence, and exit from one level to the other.
\end{abstract}

Keywords: ACCUMULATIVE FILE/ INDIVIDUAL FOLLOWING/ SYSTEMATICITY OF THE STUDENT'S DEVELOPMENTI ATTENTION TO THE DIVERSITYI PRESCHOOLER EDUCATION/ ELEMENTARY EDUCATION/ EVALUATION/

\section{Introducción}

El expediente acumulativo es el instrumento utilizado para recopilar datos referentes al desarrollo integral del educando, sus intereses, necesidades, habilidades, hábitos, aptitudes, actitudes, condiciones socio económicas del grupo familiar y otros que permiten diagnosticar y detectar progresos, potencialidades, ritmo de aprendizaje y limitaciones, con el propósito

\footnotetext{
${ }^{1}$ Master en Psicopedagogía, Licenciada en Educación Preescolar, Licenciada en Administración Educativa, los títulos de la Universidad de Costa Rica. Coordinadora de Educación Preescolar en la Sede de Occidente de la Universidad de Costa Rica. Investigadora en el Instituto de Investigación en Educación (INIE).
}

Correo electrónico: hcardena@hotmail.com

Artículo recibido: 20 de octubre, 2005

Aprobado: 12 de diciembre, 2005 
de realizar acciones de seguimiento y determinar el grado de orientación requerido. Tanto en el nivel preescolar como en I y II ciclos de la Enseñanza General Básica, la información que recopila y registra mediante el expediente acumulativo, resulta importante para definir la acción pedagógica requerida, a fin de ofrecer un proceso educativo pertinente con las características, necesidades e intereses de los niños y niñas que se atienden en los niveles citados. Por ello se propuso, mediante una investigación, analizar las funciones que cumple el expediente acumulativo de la población estudiantil en los niveles de preescolar y I y II Ciclos; y si logra satisfacer la necesidad de información de los docentes y las docentes involucradas, de manera que oriente el proceso educativo.

En este artículo se presenta el referente teórico que orienta el estudio, la metodología seguida, así como las principales conclusiones y recomendaciones. En el anexo se presenta la propuesta.

\section{El expediente acumulativo como recurso pedagógico}

\subsection{Función de la escuela}

La educación es un proceso social e individual y es importante en la humanización del hombre. La especie humana construye costumbres, normas, códigos de comunicación para la convivencia de los grupos y la especie. Así mismo, crea sistemas externos de transmisión para garantizar la supervivencia a las nuevas generaciones. A este proceso de socialización se le denomina proceso de educación. Para Denies (1992), la educación es el instrumento fundamental del progreso individual y social. A través de ella, se logra el tipo de persona que la sociedad requiere. Al respecto Gimeno (1995, p. 18) afirma: "la escuela, por sus contenidos, por sus formas y por sus sistemas de organización va induciendo paulatina pero progresivamente en los niños y las niñas las ideas, conocimientos, representaciones, disposiciones y modos de conducta que requiere la sociedad adulta".

Es evidente que a la escuela le corresponde una función social y política, porque todas las personas que la integran son sujetos actuantes que viven e interactúan en un escenario la escuela - y en él desempeñan diferentes roles mediante diversas prácticas. Así lo señala Gurdián (2000, p. 255): “Jamás la escuela puede ser una instancia separada de lo social ni de lo político, en efecto todas y todos sus actores somos portadores de posturas políticas, ideológicas y culturales determinadas".

La perspectiva de Gurdián clarifica que la función de la escuela va más allá de la transmisión de contenidos y conocimientos, precisamente cuando interpreta que todos somos portadores de cultura. Por tanto, se hace patente la necesidad de conocer esas 
particularidades de las personas con las cuales trabajamos en nuestras aulas $\mathrm{y}$, ofrecer alternativas pertinentes que cubran sus necesidades e intereses.

Para que realmente la escuela sea significativa en la vida de la persona, debe cuestionarse periódicamente, la forma de actuar del personal docente; la manera de planificar su intervención, de reaccionar ante las exigencias previstas o no de la vida cambiante del aula, y de sus creencias pedagógicas. Los educadores y educadoras tienen un rol importante que desempeñar en la formación de los educandos y en la orientación de los procesos de enseñanza y aprendizaje; factor determinante en la efectividad del currículo.

Dentro de los procesos de enseñanza aprendizaje, la información debe jugar un papel preponderante, en tanto que sirve para organizar la acción pedagógica. Por ello, se pretendió conocer la autopercepción de los docentes sobre la contribución que brinda la información del expediente acumulativo en su trabajo.

\subsection{El papel del educador y la educadora}

Los educadores y educadoras son las personas, que sin pertenecer a la familia, figuran de manera significativa en la vida del niño y niña, de ahí que su actitud, sea determinante en el desarrollo de los educandos; como lo afirma Castillejo (1992, p. 50): "una actitud sana, comprensiva y empática, pero firme y decidida, facilitará la motivación, espontaneidad y participación activa por parte de los alumnos”. Además Roffey y O’Reirdan (2004, p. 124) indica: "Todos los niños tienen necesidades de supervivencia básica, de seguridad emocional y física, de sentirse amados y atendidos, de sentirse bien consigo y de crecer y desarrollarse al máximo".

El profesorado debe desempeñar ese rol en un ambiente orientado con una guía constante, donde priven la objetividad y especialidad profesional. Además, debe ser consecuente de lo que implica poder predecir acontecimientos que tengan efecto en el salón de clase, dentro de límites razonables.

El personal docente como mediador, asume un papel crítico, dinámico, reflexivo en su actuar pedagógico, al considerar necesidades, intereses, características y experiencias del niño y la niña. De manera tal, que no es un técnico que aplica, un currículo y se basa en técnicas y estrategias elaboradas por actores externos. Cada persona y cada grupo, construye sus propios esquemas de interpretación de la realidad, a la que deben responder los y las docentes. 
Al respecto Gimeno (1995, p. 87) afirma: "la base de la eficacia docente se encuentra en el pensamiento del profesor capaz de interpretar y diagnosticar cada situación singular y de elaborar, experimentar y evaluar estrategias de intervención".

Para asumir ese papel de investigador al que se refiere Gimeno, los profesores y profesoras requieren de información que permita conocer y determinar las necesidades básicas. Para orientar el trabajo, se debe saber acerca de los conocimientos que poseen los niños y las niñas y otra información adicional que le ubique para conocer las características de cada persona.

El poseer información y sistematizarla, le permite al adulto basar sus decisiones en el testimonio de la experiencia y en el análisis de la misma. Debe desarrollar para ello, estrategias que le permitan organizar e interpretar los datos de manera apropiada, para orientar los procesos de enseñanza y aprendizaje. De esta forma, el educando es fuente primordial de información.

\subsection{El alumno y alumna: fuentes de información}

Los niños y las niñas dan las pautas para la organización del trabajo de aula. El ritmo y el avance del grupo están condicionados por factores internos y externos de la personalidad y del contexto en el cual se desenvuelven. Al respecto Gimeno (1995, p. 87) afirma: "para comprender lo que realmente sucede en los procesos de enseñanzaaprendizaje, debe tenerse en cuenta al alumno también influyen en los resultados de aquellos procesos, como consecuencia de sus elaboraciones personales".

Indistintamente del nivel con el que se trabaje, es tarea ineludible, obtener la mayor cantidad de información respecto a las particularidades, tanto grupales como individuales de sus estudiantes, con el fin de orientar de manera pertinente, la acción educativa. No se puede concretar con efectividad la planificación, ni proporcionar la atención adecuada a las diferencias individuales, si estas no son conocidas.

A través de la información recopilada, es viable dar seguimiento, en forma individual y sistemática, en cuanto a la integración social, estado de salud, aptitudes y actitudes específicas, hábitos de trabajo, sociabilidad, rendimiento, logros; a la vez debe predecir con cierto grado de certeza, la efectividad con la cual se podrán desempeñar esos niños y niñas en el futuro. Para ello, es necesario conocerles y estar muy informado de los factores que influyen en su comportamiento. 


\subsection{El expediente acumulativo}

Inicialmente, se consideró la definición de expediente acumulativo que ofrece Díaz (1995, p. 9) quien afirma: "el uso de los expedientes acumulativos o "carpetas", proporciona a todos evidencias tangibles y comprensibles y promueve la reflexión y el análisis del desarrollo de habilidades a lo largo del tiempo". Posteriormente, se recurrió a Peralta (1996, p. 153) para exponer una definición más ajustada a la realidad:

Estos registros dejan constancia de un hecho significativo protagonizado por el niño a través de una manera descriptiva lo más objetiva posible... el gran aporte que hace este tipo de registros es en el plano cualitativo, ya que es el que señala con mayor detalle todo lo que acontece respecto a un comportamiento del niño.

Sin embargo, fue necesario enriquecerla hasta llegar a la siguiente definición adaptada: Es el instrumento utilizado, para recopilar datos referentes al desarrollo integral del educando, sus intereses, necesidades, habilidades, hábitos, aptitudes, actitudes y otros que permitan diagnosticar y detectar progresos, potencialidades, ritmo de aprendizaje y limitaciones, con el propósito de realizar acciones de seguimiento y determinar, el grado de orientación requerido. Se emplea para recopilar y actualizar en forma ordenada la información. Acumulativo porque es un acervo de datos, que ofrece una visión global y objetiva de la persona.

Por su parte, Knapp (1983), establece que el expediente acumulativo es un medio para un fin, nunca un fin en sí mismo, y tiene valor únicamente cuando sirve a las necesidades de los niños y de las niñas.

Esta nueva definición agrega un ingrediente fundamental: el fin del expediente acumulativo. Es decir, especifica el valor del expediente como recurso pedagógico para el docente, el cual le ayuda a identificar y a comprender las necesidades individuales de los niños y las niñas.

Así, se concibe el expediente acumulativo como un recurso que provee la información necesaria; la continuidad es de gran valor, por cuanto permite, entender el comportamiento de los educandos; descubrir sus necesidades individuales; distinguir entre tendencias pasajeras y estables en su conducta, y descubrir los factores causantes de sus posibles dificultades. 
Además, ayuda al personal docente a concienciar a los padres y a las madres para que tengan una noción más objetiva y precisa de las acciones, aptitudes especiales y necesidades concretas de los y las infantes.

\section{Metodología}

Se elaboró un estudio descriptivo para analizar las funciones que cumple el expediente acumulativo del niño y la niña en el nivel de educación preescolar y de I y II ciclo de la EGB de la Dirección Regional de Educación de Cartago.

Se trabajó con tres grupos de profesionales, el primer grupo estuvo conformado por 20 docentes del nivel preescolar, con experiencia en jardines de niños independientes y dependientes. El segundo grupo, se integró con 20 profesores y profesoras de I y II Ciclos, que tienen experiencia en el manejo de expedientes acumulativos, y el tercer grupo lo conformaron 20 docentes pertenecientes a los comités de orientación por II ciclo de Educación General Básica, quienes son los responsables de dar seguimiento a la aplicación de los expedientes acumulativos, con estos grupos se trabajó la técnica de grupos focales.

Posteriormente, se aplicó un cuestionario que condujo a profundizar en las percepciones que los docentes y las docentes tienen sobre su quehacer en relación con los expedientes acumulativos.

Asimismo se elaboró una guía para analizar los expedientes acumulativos aplicados a los educandos.

\section{Resultados}

Los resultados se presentan en dos apartados a saber, percepciones del personal docente sobre el expediente acumulativo y estrategias que aplica el profesorado en el uso del expediente acumulativo.

\subsection{Percepciones del personal docente sobre el expediente acumulativo}

En relación con la definición de expediente acumulativo las personas participantes, lo ubican, en su mayoría, como un instrumento que permite obtener información, criterio que coincide con la teoría, cuando se afirma que es un instrumento para "recopilar y actualizar, en forma ordenada, la información individual y grupal que se va obteniendo de los niños y niñas, a través de diferentes medios para diagnosticar su desarrollo integral". 
Los integrantes de los comités de orientación, así como las docentes de educación primaria y preescolar, consideraron que las instrucciones para usar el expediente acumulativo facilitaron su aplicación, al ser claras, sencillas y precisas.

Sobre la finalidad de los datos recopilados en este expediente, las participantes señalan las limitaciones y habilidades de niños y niñas para un trato individual. Estas respuestas coinciden con la finalidad del expediente acumulativo: realizar acciones de prevención, referencia, tratamiento y seguimiento, a partir de un conocimiento más objetivo de cada niño y niña.

Una mayoría de docentes de Educación Preescolar, destaca que el conocimiento de las necesidades, los intereses y el ritmo de aprendizaje, le ha permitido hacer las adecuaciones curriculares. Las y los docentes de I año e integrantes de Comité de Orientación, coinciden en que la información ayuda a orientar psicológica e intelectualmente al educando, así como plantear estrategias de carácter interdisciplinario para la atención individual. Estas respuestas denotan que el expediente acumulativo, constituye un recurso muy valioso en los procesos de enseñanza y aprendizaje.

Se encontró que la mayoría de las docentes de I y II ciclos actualizan el expediente acumulativo trimestralmente, en tanto las docentes de preescolar lo actualizan con más regularidad, situación que demuestra en el personal de preescolar, mayor constancia de actualización. El expediente acumulativo debe estar actualizado y debe mantener la información que oriente la actuación o el procedimiento en el aula en forma permanente. Pereira (1981) fundamenta esa premisa, al señalar como característica fundamental del expediente acumulativo, la continuidad en la forma, el contenido y el balance de la información.

En la aplicación de los instrumentos, algunos participantes reconocen que el aporte del expediente acumulativo, es de gran utilidad, pues les permite diagnosticar aspectos psico-sociales; llevar un seguimiento sistemático del progreso del educando; referir casos a especialistas y presentar mayor información al personal que atenderá a los niños y niñas posteriormente. Estas respuestas son congruentes con los objetivos para los cuales fueron creados los expedientes acumulativos que señalan, entre otros, entender el comportamiento de los alumnos y las alumnas; descubrir sus necesidades individuales; distinguir entre tendencias pasajeras, y descubrir los factores causantes de sus posibles dificultades. 


\subsection{Estrategias que aplica el profesorado en el uso de los expedientes acumulativos}

Ante la ausencia de políticas definidas en el nivel nacional, en relación con el uso del expediente acumulativo, el personal docente ha aplicado algunas iniciativas individuales que conducen a facilitar su uso, como vía para fortalecer el trabajo con los niños y niñas que tenían a su cargo. Algunas de esas estrategias, según los datos obtenidos fueron:

“... realizo revisiones trimestrales” (comité de orientación).

"Yo realizo entrevistas con padres y madres, observación y evaluación".

"Realizo reuniones con el personal para concientizarlos de la importancia de llevar un control del desarrollo evolutivo de los educandos".

"Yo personalmente fotocopio los instrumentos".

“... cada maestra se encarga de mantener los expedientes de su grupo y de ponerlos en práctica".

"Los lleno durante el año y se pasan al iniciar el siguiente curso lectivo".

"Uso planes remediales".

Estas iniciativas demuestran el interés del personal docente por el acceso a la información y aunque no resuelven el problema de la falta de sistematización en el nivel nacional, se convierten en un aporte para promover el uso del expediente acumulativo, con el propósito de ofrecer el seguimiento adecuado. Es evidente que las docentes de preescolar sistematizan, con mayor regularidad, el expediente acumulativo en tanto las de primaria no. Estas últimas aducen que es extenso, repetitivo y requiere de mucho tiempo para completar la información. Estos resultados motivan la elaboración de una propuesta sencilla y funcional de expediente acumulativo.

Así, en términos generales, se puede afirmar que hay un reconocimiento entre las personas participantes respecto de la relevancia de la información que se recopila mediante del expediente acumulativo, y su compromiso en la sistematización de los datos, pero a su vez, señalan desventajas en la aplicación del expediente acumulativo. Sobre todo las docentes del nivel preescolar se quejan de que el trabajo realizado durante un año, es obviado por una gran cantidad de docentes del I año escolar. Han encontrado expedientes completos tirados en el basurero o, simplemente, no los utilizan como recurso para planificar su acción pedagógica. Es decir, no se les da continuidad, ni seguimiento. Algunas docentes de I y II ciclos alegan falta de tiempo, un espacio estructural insuficiente; otras lo toman como imposición y más trabajo. 
Otro aspecto que se logró deducir en el trabajo con los diferentes grupos a través de expresiones y preguntas de las participantes, es que el instructivo del expediente acumulativo es desconocido por una parte de los docentes y las docentes, pues algunos y algunas han llenado el instrumento, sin conocer el instructivo; lo que afecta, por supuesto, su aplicación.

\section{Algunas conclusiones}

El estudio llevado a cabo en la Dirección Regional de Educación de Cartago, permite obtener una amplia visión del comportamiento de las docentes del nivel preescolar, de I y II Ciclos y comités de orientación, con respecto al uso del expediente acumulativo, como recurso pedagógico que permite obtener información para orientar los procesos de enseñanza y aprendizaje. En este sentido, se deduce que los sujetos que participaron en el estudio, muestran un dominio incipiente de las habilidades para usar el expediente acumulativo, sobre todo, en su continuidad en los ciclos siguientes.

Adicionalmente, como resultado del estudio, se ha llegado a las siguientes conclusiones:

1. Todos los sujetos reconocen la importancia del expediente acumulativo como instrumento que les permite obtener información, diagnosticar y realizar acciones de seguimiento.

2. El total de docentes de educación preescolar, opinaron que con la aplicación de los expedientes acumulativos, obtuvieron una información precisa, global y actualizada. Además, la mitad opinó, que el control, seguimiento individual y sistemático fue otro logro, compartido por la población docente de I año. Asimismo la población correspondiente a los comités de orientación señalan como logros la posibilidad de diagnosticar y detectar problemas de aprendizaje; señalamiento con el que coincide una tercera parte de docentes de I año.

3. Es generalizada la percepción de los sujetos acerca de la necesidad de incluir un apartado de "datos generales", más información del hogar y aumentar la información del historial clínico, en el "apartado de salud", lo que hace suponer que falta claridad en cuanto a la información mínima.

4. Se indican como limitaciones en el uso del expediente acumulativo, el espacio estructural insuficiente, la no continuidad en ciclos posteriores, así como la poca disponibilidad de los padres y madres de familia para brindar la información requerida. Los integrantes de los comités de orientación encontraron como mayor 
limitación, la poca importancia que las educadoras y los educadores le dan a los expedientes acumulativos.

\section{Recomendaciones para mejorar el expediente acumulativo del niño y la niña, como recurso pedagógico}

Algunas sugerencias para mejorar el expediente acumulativo del niño y la niña, como recurso pedagógico en el nivel preescolar y I y II ciclos de la Educación General Básica, de acuerdo con los resultados de la investigación, son las siguientes:

\subsection{Dirección y Comités de orientación}

- Ofrecer la inducción a los educadores y las educadoras, mediante talleres sobre la aplicación del expediente acumulativo

- El director o directora le facilitará a cada docente, al principio del curso lectivo, un instructivo y tantos expedientes acumulativos, como alumnos y alumnas ingresan para su debida sistematización.

- El lugar donde se mantienen los expedientes acumulativos reunirá condiciones de seguridad, limpieza y funcionalidad. Además, es necesario que se mantengan al alcance de los educadores y las educadoras en el momento que los requieran, siguiendo un orden lógico de archivo

- El expediente acumulativo se inicia con el ingreso del estudiante al jardín de niños y se le da continuidad sistemática de un nivel a otro y de una institución a otra, incluyendo aulas integradas y otros servicios de educación especial.

- El expediente acumulativo se entrega de docente a docente, a través del comité de orientación, o de director a director cuando hay traslado, evitando el pase directo a las familias.

- El director y la directora deben procurar la consulta y utilización del expediente acumulativo por parte de los docentes y las docentes de cualquier especialidad que atienden al educando, de lo contrario carecerá de valor. 
- Los comités de orientación brindarán seguimiento al expediente acumulativo con una frecuencia semestral como mínimo y lo revisará al finalizar el curso lectivo para determinar su actualización.

- Se debe mantener los expedientes acumulativos a disposición de los comités de apoyo.

- Como parte de la información adicional se contempla:

- Hoja de pre-matricula y matrícula

- Fotocopia de constancia de nacimiento

- Fotocopia de tarjetas de vacunas

- Historial del desarrollo del educando y socio-económico de la familia

- Diagnóstico inicial y como resultado el Perfil de entrada del educando

- Pruebas, test, escalas aplicadas y su respectivo análisis, entre otras:

- Agudeza visual (Test Snellen)

- Agudeza auditiva

- Lateralidad

- Test Esquema Corporal

- Escala de E.D.I.N

- Otras que considere pertinente según caso particular que amerite el estudiante

- Anotaciones de comentarios recibidos del hogar con respecto al alumno o alumna.

- Cualquier dibujo, collage u otro que aporte información relevante

- Hoja de adecuaciones curriculares

- Registro anecdótico

- Visitas de madres, padres o encargados a la institución y del docente al hogar

- Entrevista al educando

- Planes de desarrollo aplicados con los respectivos resultados

- Perfil de salida del educando (refiérase a los programas de estudio de cada nivel)

- Copia de referencia para algún, especialista o servicio especial (psicología, trabajo social, terapia, otros)

- Copias de valoraciones realizadas por especialistas, cuando el educando ha sido referido 
- Otros (copia de documentos correspondientes a adecuaciones curriculares)

\subsection{Docentes}

- Todo educador y educadora que haga uso del expediente acumulativo debe leer previamente el instructivo.

- El expediente acumulativo se organiza en una carpeta en buen estado, que contenga instrumento con la información solicitada y la adicional, encabezada por un índice de acuerdo con la numeración y el orden que lleva cada documento.

- Desde el inicio del curso lectivo, la información debe mantenerse actualizada e ir incorporando paulatinamente los progresos, respetando la información adicional de años anteriores.

- Tenga en cuenta que aunque el expediente acumulativo es un instrumento que contribuye al conocimiento de la niña y el niño, el contacto personal y permanente discente - docentes es insustituible en este proceso evolutivo, pues este no registra la evolución total.

- Las anotaciones en el expediente acumulativo deben ser claras, precias y concisas, de tal manera que expresen el máximo de circunstancias con el mínimo de palabras.

- La información básica se solicita a todos los infantes individualmente, pero posee un amplio margen de flexibilidad para que incorpore otros datos que considere pertinente y de relevancia a cada niño y niña.

- La información debe procurar un equilibrio con respecto a las áreas de desarrollo, sin descuidar ninguna apreciación en cuanto a las fortalezas y limitaciones que posean los educandos.

- Toda información debe basarse en conductas observables. Cuando tenga duda de alguna situación especial del niño y la niña refiérase al comité técnico, a fin de evitar anotaciones que trasciendan su campo de acción. 
- La información suministrada es confidencial por parte del educador o educadora así como por personas especialistas que hagan uso del mismo. Alguna referencia requiere ser analizada en forma privada, con el propósito de evitar malas interpretaciones de personas que no posean la comprensión del nivel de desarrollo que alcanza el educando.

- La información debe ser secuencial y con anotaciones cronológicas, según los datos obtenidos.

- Es necesario distinguir los hechos y datos concretos (objetividad) de las opiniones personales, así como también cuidar el lenguaje escrito que se refiera a situaciones psicosociales particulares del niño, la niña y su familia.

- Al final se debe incluir una crónica general que describa el progreso y desarrollo del niño y la niña a lo largo del curso lectivo, basado en la información recopilada y en el perfil de salida.

- Se deben entregar los expedientes al comité de orientación de la institución para la revisión final. Los mismos se mantendrán en el lugar de seguridad dispuesto por la Dirección, para la continuidad al año siguiente.

A partir de las conclusiones a que se llegó, se diseñó una propuesta que facilitara la aplicación del expediente acumulativo en el nivel preescolar y I y II ciclos de la Educación General Básica, la cual se anexa a este artículo.

\section{REFERENCIAS}

Ary, D., Jacobs, L. y Razaviech, A. (1997). Introducción a la investigación pedagógica. México, D.F.: Trillas.

Bassedas, C. y otros. (1990). Evaluación y seguimiento en parvulario y ciclo inicial. España: Aprendizaje Visor.

Carretero, M. (1997). El aprendizaje significativo, la importancia de los saberes previos y la motivación de los alumnos. Revista Zona Educativa, Vol. 14. Argentina: Ministerio de Cultura y Educación. 
Chavarría, M.C. (1991). Guía de observación y evaluación de Centros Infantiles. Costa Rica: Editorial Universidad de Costa Rica.

Denies, C. (1992). Didáctica del nivel inicial. Barcelona: El Ateneo.

Dirección Regional de Cartago. (1993). Expediente del alumno preescolar, I y II Ciclos. Asesorías Educación Preescolar y Orientación. Costa Rica: Ministerio de Educación Pública.

Gimeno, S. (1995). Comprender y transformar la enseñanza. Madrid: Morata.

Gurdián, A. (2000). Una mirada crítica a la educación. Costa Rica: Editorial de la Universidad de Costa Rica.

Hatch, R. y Costar, J. (1978). Actividades de orientación en la escuela primaria. México: Limusa.

Knapp, R. (1983). Orientación escolar. España: Morata.

Marí, R. (2001). Diagnóstico pedagógico. España: Ariel Educación.

Ministerio de Educación Pública (MEP) - Costa Rica. (2003). Elaboración y utilidad del expediente acumulativo del estudiante del ciclo de transición en la educación preescolar y en el primer año de la educación general básica. San José: MEP.

Peralta, V. (1996). El currículo en el jardín infantil. Chile: Andrés Bello.

Pereira, M.T. (1981). Orientación del estudiante de educación primaria. San José: EUNED.

Rojas, M. (1996). Expediente del alumno preescolar I y II Ciclo. Revista Preescolar. Volumen 10: 25-29. Costa Rica: Ministerio de Educación Pública.

Rojas, M. y Cárdenas, H. (1998). El Expediente Acumulativo en el Nivel Preescolar, y la Educación General Básica en la Dirección Regional de Educación de Cartago. Tesis de Licenciatura, Universidad de Costa Rica, Costa Rica. 


\begin{abstract}
ANEXO
Propuesta: El expediente acumulativo en educación preescolar y I y II Ciclos de la Educación General Básica.

La propuesta surge a partir de los resultados de un proceso de investigación titulado "El expediente acumulativo en el nivel preescolar y la educación general básica de la Dirección Regional de Cartago". La misma pretende ofrecer un instrumento de expediente acumulativo con información relevante, que les permita al personal docente de preescolar y I y II Ciclos, orientar, adecuadamente, los procesos de enseñanza y aprendizaje.

Es importante señalar que el estudio en general y la propuesta en particular, sirvieron de sustento para una política nacional en relación con la continuidad del expediente acumulativo, que se dio a conocer, mediante el documento "Elaboración y utilidad del expediente acumulativo del estudiantes del ciclo de transición en la educación preescolar y en primer año de la educación general básica (2003).
\end{abstract}

\title{
Descripción general de la propuesta
}

El conocimiento psicosocial del alumno, no puede dejarse a la memoria del personal docente; sobre todo, si tomamos en cuenta que estos funcionarios tienen a su cargo un gran número de estudiantes y que, a menudo, cambian de lugar de trabajo. Por este motivo, los datos deben quedar recopilados en forma ordenada y secuencial, para ser utilizados en beneficio del estudiante en el momento preciso y en forma profesional.

\section{Objetivos del expediente acumulativo}

1. Conocer las diferencias individuales en cuanto a: condiciones socio-económicas del grupo familiar, condiciones de salud y rendimiento escolar del alumno.

2. Realizar acciones de prevención, referencia, tratamiento y seguimiento a partir de un conocimiento más objetivo del niño o la niña.

3. Adecuar las experiencias de aprendizaje a las necesidades, intereses y expectativas de los estudiantes y las estudiantes.

4. Obtener una noción más objetiva y precisa de las realizaciones, aptitudes y necesidades del alumno para informar a las familias. 
5. Suministrar datos oficiales relativos a la asistencia, resultados, promoción y progresos del educando.

6. Recopilar y actualizar en forma ordenada la información que se va obteniendo de cada niño y niña a través de diferentes medios a lo largo de su desarrollo evolutivo.

\section{Instructivo para la aplicación del expediente acumulativo}

El expediente acumulativo desde preescolar hasta I y II Ciclos, consta de cuatro apartados que permitirán recopilar la información básica para brindar el seguimiento individual que requieren el niño y la niña.

\section{Datos generales}

Comprende información general del estudiante y del grupo familiar. Cuando el encargado del niño o la niña es otra persona que no sea su padre o madre, se debe indicar debajo del nombre, el nexo que tiene con el alumno.

Al final de esta sección, aparece un espacio de observaciones en el cual el personal docente anotará aquellos cambios importantes que se operen en la familia, tales como: separación, divorcio, muerte, accidentes. Además se debe definir quién es la persona encargada del niño o la niña, el número de miembros que viven en la casa, ocupación, oficio, etc. así como la problemática familiar en el nivel social, psicológico y económico. Es preciso indicar el espacio según el nivel o año que atiende y respectiva fecha en que se hace la observación.

Se debe tener en cuenta que la información específica de la familia se registra mediante una anannesis y que los datos que corresponden a un estudio socioeconómico que se adjunta en la información adicional, por lo que no son contemplados en el esquema propuesto.

\section{Datos acerca de la salud del niño y la niña}

Se trata de determinar en los niños y las niñas, el origen probable de trastornos de su salud, así como las acciones preventivas y de atención que se han brindado.

2.1- Valoración nutricional PESO-TALLA: es el indicador del estado nutricional actual del niño o la niña. Está clasificado según el Ministerio de Salud, de la siguiente manera: 
Obesidad, normal, desnutrición leve, desnutrición moderada y desnutrición severa, categorías que se aplican, según la gráfica PESO PARA TALLA-NIÑOS-NIÑNS.

Valoración inicial: Es la condición en que ingresa el niño (a), lo que permitirá que el docente o la docente pueda aplicar algunas acciones.

Valoración media: Permitirá el seguimiento de acciones ejecutadas, a partir de la valoración inicial.

Valoración final: Demostrará si las condiciones se han mantenido, disminuido o mejorado, según sea el caso.

Se recomiendan las Guías alimentarias para la educación nutricional en Costa Rica. 1998.

2.2- Enfermedades padecidas por el niño, la niña y la familia: Se debe incluir además de las enfermedades físicas o mentales (sarampión, varicela, paperas, rubéola, escarlatina, hepatitis, tifoidea, tos ferina, depresión, autismo, esquizofrenia, paranoia), otras que han padecido o padecen, tales como: enfermedades de la piel, convulsiones, hipertensión, diabetes, neumonía, infecciones de oído, bronquitis, asma, anginas, catarro, amigdalitis, fiebre reumática, infecciones urinarias, temperaturas altas, desnutrición (grado). Se debe indicar en el espacio, respectivo, si recibe tratamiento, de qué tipo y con qué frecuencia.

2.3- Pruebas aplicadas, test Snellen, auditivo, lateralidad; psicológicas y neurológicas, si es el caso, pedagógicas, otras con sus respectivos resultados y fecha de aplicación por especialistas.

2.4- Manifestaciones de conductas relevantes en el niño o niña, tales como: hiperactividad, agresividad, timidez, atención dispersa, aislamiento, fobias, temores. Además, se deben incluir aquellas conductas producidas por consecuencia de enfermedad o por tratamiento farmacológico.

\section{Desarrollo evolutivo y rendimiento escolar}

Se pretende obtener una visión integral del desarrollo evolutivo del niño y de la niña desde el nivel preescolar hasta sexto año. 
3.1- Cuadro referente a educación preescolar: debe indicar el resultado cualitativo anual, obtenido por el niño o la niña en las tres áreas de desarrollo. En este apartado, debe aparecer un resumen que describa el trabajo realizado con esa persona para que el personal docente que lo recibe, pueda darle continuidad.

La información anual sobre ausentismo se hace a manera de reporte, cuando es muy frecuente y se debe anotar los motivos.

3.2- Cuadro referente a l y II ciclos: se anotará información anual del ausentismo presentado por el niño y la niña y el promedio anual obtenido, tanto en las diferentes asignaturas, como en conducta.

Es importante que anote si ha aprobado o ha reprobado el curso lectivo.

\section{3- Cuadro relacionado con aptitudes, intereses y otras manifestaciones observadas} en preescolar, I y I/ ciclos: El docente o la docente anota en el espacio correspondiente en forma resumida, la apreciación del proceso de desarrollo del niño o la niña en aquellas áreas en que se destaca: aptitudes e intereses especiales, valores, hábitos que ha manifestado dentro y fuera del aula, así como algunas limitaciones presentadas. Una información que lleve al docente y la docente a una investigación cualitativa o grupal, que permita fortalecer y satisfacer potencialidades y necesidades de los educandos en el proceso de enseñanza y aprendizaje.

\section{Información adicional}

Se trata de rescatar la información recopilada por el profesorado durante el curso lectivo, por medio de diagnósticos, entrevistas, anannesis, visitas al hogar, observaciones, registros anecdóticos, tarjeta de vacunas, otros instrumentos utilizados y acciones desarrolladas, con el fin de contemplar el resumen brindado en el expediente y proporcionar mayor cantidad de aspectos útiles al docente, para favorecer los procesos de enseñanza y aprendizaje.

Se debe adjuntar el informe de otros especialistas que han atendido al niño o niña (terapista emocional, terapista de lenguaje, psicólogo y otros). 
Se puede anotar lo que se considera pertinente en el espacio "otras anotaciones", así como anotar información sobre maltrato, abuso físico, sexual o psicológico, drogadicción, otros.

Nota:

Al ser éste un expediente acumulativo, es importante que los docentes y las docentes utilicen el espacio de manera razonable y que tomen en cuenta que otros educadores y educadoras también harán uso de esos espacios. 